\title{
Title: Comparison of Material Activity and Selectivity in the Electrocatalytic Oxidation of Dibenzothiophene
}

Author Names: Victoria Kompanijec and John R. Swierk ${ }^{\mathrm{z}}$

Affiliation(s): Department of Chemistry, Binghamton University, Binghamton, New York 13902, United States

zjswierk@binghamton.edu

\begin{abstract}
Due to adverse effects of sulfur-containing compounds present in fuel oils, there is an increasing demand for efficient methods of removing sulfur from oil products, such as oxidative desulfurization. In this work, a set of five materials (gold, glassy carbon, nickel, palladium and platinum) were evaluated as electrochemical catalysts for the oxidation of DBT. Electrolysis at $1.58 \mathrm{~V}$ was performed without water present (producing DBT dimer) and with the addition of $2 \mathrm{M}$ water (producing DBTO). LC-MS and NMR were used to characterize the oxidation products. It was found that Faradaic efficiencies ranged from $18.4-56.5 \%$ for consumption of DBT without water present, and there was a correlation between higher rate constants, lower activation energies and more efficient DBT oxidation. After the addition of water, the formation of DBTO had highest selectivity when catalyzed by gold, with a Faradaic efficiency of $87.9 \%$. Group ten metals demonstrated low Faradaic efficiencies due to competitive water oxidation. Though there were differences in the observed selectivity for DBT oxidation, all catalysts reduced the concentration of DBT in solution by similar amounts. Of the materials tested, gold was the most selective for oxidation to DBTO, with the presence of water improving the overall reaction activity.
\end{abstract}

\section{Introduction}

The presence of sulfur-containing organic compounds in fuel oils leads to the formation of environmentally harmful sulfur oxides $\left(\mathrm{SO}_{\mathrm{x}}\right)$ upon combustion. ${ }^{1}$ Exposure to these products can lead to acute respiratory distress and long-term chronic lung illness in humans and animals, and they contribute to the formation of acid rain given sufficient accumulation. ${ }^{2}$ In addition, $\mathrm{SO}_{\mathrm{x}}$ production in engines also results in the degradation of catalytic convertors in vehicles. ${ }^{3}$ Because of these adverse effects, restrictions on the sulfur content in commercially available petroleum products have been imposed since the 1990s, and restrictions have continued to become more stringent across the globe in recent years. ${ }^{4,5}$ The leading industrial method of removing sulfur from fuel oils is thermal hydrodesulfurization (HDS). In this refining process, a feedstock of naphtha is added to a fixed-bed reactor containing an alumina-based catalyst, typically doped with a CoMo and/or NiMo catalyst. A stream of hydrogen-rich gas is pumped 
into the system, facilitating the hydrogenation of the $\mathrm{C}-\mathrm{S}$ bonds, forming instead $\mathrm{C}-\mathrm{H}$ and $\mathrm{S}-\mathrm{H}$ bonds. ${ }^{6}$ HDS has been shown to be effective at reducing sulfur content to the levels required by governing agencies in the past, but it is lacking in several areas. First, HDS has intense energy requirements, as the process must be performed at high temperatures and low-pressure conditions to be thermodynamically viable. Another issue is that unfavorable side products such as $\mathrm{H}_{2} \mathrm{~S}$ are formed during HDS, and an extra step must be implemented to remove this compound before the oil can be processed for distribution. ${ }^{7}$ Finally, HDS is not effective at removing certain types of sulfur compounds, specifically thiophenes and their alkylated derivatives. These molecules contain flat, aromatic rings and sterically prevent HDS catalysts from interacting with the sulfur atom. ${ }^{8}$ This creates a lower limit for the sulfur content able to be removed through treating fuel with HDS. As a result, there is significant interest in alternative methods of petroleum desulfurization, with one promising option being oxidative desulfurization (ODS).

ODS is a method of sulfur removal which involves oxidizing sulfur impurities within the oil, thereby creating a polar compound that can be easily separated from the nonpolar environment. There are several different approaches to achieve ODS, including adsorptive oxidation using porous materials, ${ }^{9}$ biomimetic techniques modelling the pathways of oxidation-mediating enzymes, ${ }^{10}$ and photo- and electrocatalysis. ${ }^{11,12}$ Electrochemical ODS is a promising area of interest because it can be performed under mild conditions and there is a high degree of tunability available through electrode choice. ${ }^{13}$ In 1983, Lalvani et al. demonstrated an electrochemical method for oxidizing metal sulfides and sulfoxides, as well as sulfur-containing organics, including thiophene. ${ }^{14}$ The group used either a platinum or graphite working electrode in a solution of aqueous $\mathrm{KCl}$ mixed with coal. Under these conditions, it was shown that up to $40 \%$ of the total sulfur content could be extracted from the coal. This was one of the first experiments which utilized electrocatalytic oxidation as route to removing sulfur-containing compounds from solution. A patent by Ahonen demonstrated an electrolysis cell containing platinum coated electrodes to oxidize and remove sulfur from heavy fuel oils containing approximately $2 \%$ sulfur by weight. ${ }^{15}$ The reaction conditions were believed to result in anionic polymerization of thiophenes, which could then be phase separated from the emulsion, reducing the sulfur content of the oil to 0.15 $0.83 \%$ by weight. In the years since these preliminary studies established the possibility of electrooxidation of sulfur compounds found in fuel oils, there has been a surge in reports of electrochemical systems designed to optimize the oxidation reaction. A study in 2007 conducted by Wang et al. began to tighten the focus of oxidation to heterocyclic compounds such as thiophene and its derivatives, which are the most difficult to remove from fuel oils. ${ }^{16}$ The working electrode, cerium dioxide supported on activated carbon, was chosen due to its large positive metal potential, and exhibits the increasing complexity of catalyst design over time. An important thing to note about this study is that 
the catalysis was attributed to the activity of $\mathrm{Ce}^{3+}$ ions in the electrolyte rather than a direct oxidation on the surface of the electrode. In this experiment a secondary oxidant (e.g., hydrogen peroxide or functionalized silica) was not used. However, later electrocatalytic systems skewed heavily toward the inclusion of a compound to make oxidation more accessible. ${ }^{17}$ Because there is so much variation in electrochemical parameters, it is difficult to accurately compare the performance of different working electrodes in existing studies of electrochemical oxidation. Emphasis on seeking a high-performance catalyst has yielded impressive results for oxidation of dibenzothiophene (DBT), a model refractory thiophene typically found in crude oils. Nanocomposite catalysts developed in recent years, such as CTAB-PTA@CS and TBAPMo ${ }_{11} \mathrm{Cu} @ \mathrm{CuO}$, have been shown to have a DBT conversion of 95\% and 98\% upon one hour of electrolysis respectively. ${ }^{18,19}$ Materials based on group four metals such as $\mathrm{TiO}_{2}$ and $\mathrm{ZrP}$ demonstrate the ability to remove nearly all DBT under optimal conditions. ${ }^{20,21}$

Despite these developments, little is known about what specific material parameters play a role in selectivity and activity for the oxidation of sulfur-containing compounds. In this study, we characterize a series of common electrode materials for electrocatalytic DBT oxidation, specifically gold (Au), glassy carbon (GC), nickel (Ni), palladium (Pd), and platinum (Pt), and compare the overall electrocatalytic activity with kinetic parameters. We determined the activation energies and Faradaic efficiencies of these materials, both in dry conditions and in an environment of $2 \mathrm{M}$ water, demonstrating multiple oxidation pathways and kinetic results. The results of our work show a strong selectivity for DBT oxidation can be obtained for electrodes with a low activation energy for DBT oxidation and a high activation energy for water oxidation. In addition, Au electrodes exhibit anomalously high activity for DBT oxidation because of a higher local concentration of a DBT at the electrode, which we attribute to an increased interaction between sulfur and $\mathrm{Au}$.

\section{Experimental}

\section{Materials}

Chemicals, including dibenzothiophene (DBT, 98\%), dibenzothiophene sulfone (DBTO2, 97\%) and ammonium hexafluorophosphate (NH4PF6, 99.98\%), were purchased from Sigma Aldrich and used with no further purification. Dibenzothiophene 5-oxide (DBTO) was purchased from Santa Cruz Biotechnology. Au, GC, Pd, and Pt rotating disk electrodes (RDE) with a surface area of $0.25 \mathrm{~cm}^{2}$ were purchased from Pine Research. A nickel film was deposited for one hour on the gold rotating disk electrode using the Watts formulation, ${ }^{22}$ specifically in an aqueous solution of $250 \mathrm{~g} / \mathrm{L} \mathrm{NiSO}_{4}, 50 \mathrm{~g} / \mathrm{L}$ $\mathrm{NiCl}_{2}, 40 \mathrm{~g} / \mathrm{L} \mathrm{H}_{3} \mathrm{BO}_{3}$, using an $\mathrm{Ag} / \mathrm{AgCl}$ reference electrode and Ni counter electrode while passing a 
constant current density of $-12.5 \mathrm{~mA} / \mathrm{cm}^{2}$.

\section{Experimental Conditions}

All electrochemical experiments were performed within a rotating disk electrode (RDE) glass cell with a water jacket and controlled by a potentiostat (SP-50 BioLogic). Before testing, the working electrodes were polished using diamond polishes at 15, 3, $1 \mu \mathrm{m}$ and a $0.05 \mu \mathrm{m}$ alumina polish (BASi) and then washed with water, acetone, and dried. The Au and Pt electrodes were also electrochemically polished using cyclic voltammetry $(\mathrm{CV})$ from $-0.3 \mathrm{~V}$ to $1.2 \mathrm{~V}$ in $0.5 \mathrm{M} \mathrm{H}_{2} \mathrm{SO}_{4}$. The counter electrode used was a coiled platinum wire placed in a fritted glass isolation tube. A silver pseudo-reference electrode was used during all experiments and was determined to have an operating potential of $40 \mathrm{mV}$ vs. NHE at room temperature. The electrolyte solution was a $0.1 \mathrm{M}$ solution of $\mathrm{NH}_{4} \mathrm{PF}_{6}$ in acetonitrile, which was dried over $4 \AA$ molecular sieves for 24 hours prior to use. For wet experimental conditions, $2 \mathrm{M}$ ultrapure (18.2 $\mathrm{M} \Omega$ ) deionized water was added to the dry electrolyte. These experimental parameters were modelled after those reported by Méndez-Albores et al., as they demonstrated clear oxidation of DBT under mild conditions with an undecorated GC electrode. ${ }^{23}$ All CV and linear sweep voltammograms (LSV) were performed at a rate of $20 \mathrm{mV} / \mathrm{s}$. The electrolyte was not purged free of oxygen because electrochemical tests under air-free conditions yielded no appreciably different results. Rotational studies were performed using a Pine Research Modulated Speed Rotator. Electrochemical data was recorded and analyzed using the software EC-Lab Version 11.21. Temperature controlled experiments were carried out via water recirculation (PolyScience 6-liter Analog Controller Refrigerated/Heated Circulating Bath), and the reference electrode potential was determined at every temperature using ferrocene as a standard.

Chronoamperometric bulk electrolysis was carried out at $1.58 \mathrm{~V}$ vs. SHE and was stopped once the current-time plot reached a plateau (approximately 1 hour). Aliquots of the resulting solutions were diluted by a factor of ten before being filtered for quantitative LC-MS testing (Shimadzu LC-MS 2020), while a fraction was dried under vacuum and dissolved in $\mathrm{CD}_{3} \mathrm{CN}$ for qualitative proton NMR (Bruker Avance III HD 400MHz).

\section{Results and Discussion}

\section{Electrochemical Analysis in Dry Conditions}

Initial CV testing confirmed the presence of an irreversible oxidation peak for DBT at approximately $1.58 \mathrm{~V}$ vs SHE, as shown in Figure 1. Peaks measured on electrodes fell within a $200 \mathrm{mV}$ range of this value. This agrees with the oxidation potential of DBT to DBTO reported by Méndez-Albores et al. in the 
presence of water and without the use of a secondary oxidant such as $\mathrm{H}_{2} \mathrm{O}_{2} .{ }^{21}$ Water has been shown to be a vital component in the oxidation pathway of DBT to its corresponding sulfoxide and sulfone. However, when analysing electrocatalysts for DBT oxidation, the DBT oxidation peak can be obscured by water oxidation. ${ }^{24}$ Because of this, initial electrochemical experiments were performed in the absence of water. Upon bulk electrolysis, the major oxidation product for all electrodes was found to be a dimer of DBT, confirmed through NMR and LC-MS analysis (Fig. S2; S3, ESI). This is consistent with the major oxidation product identified by Bontempelli et al. after electrolysis of DBT at $1.6 \mathrm{~V}$ in an electrolyte containing no water. ${ }^{25}$ The DBT dimer has a similar polarity to DBTO and $\mathrm{DBTO}_{2}$, as demonstrated by its liquid chromatography retention time, meaning that it would also be separated from DBT in a polar solvent wash. There is no DBTO or $\mathrm{DBTO}_{2}$ formed in the absence of water, as shown by the NMR and LC-MS spectra of the post-electrolysis solution (Fig. S2; S3, ESI). Assuming that the dimer is formed via coupling between two cation radicals in a similar process to the oxidative polymerization of a thiophenebased oligomer measured by Audebert et al., it can be inferred that the process occurring at $1.58 \mathrm{~V}$ is a one electron EC step. ${ }^{26}$ The resulting Faradaic efficiencies were calculated using LC-MS (Fig. S4) of DBT consumption and are listed in Table 1.

To gain kinetic information about DBT oxidation under these conditions, Koutecký-Levich analysis was performed. The distortion of the cyclic voltammogram shape at varying rotational rates allows the standard first order rate constant $\left(k^{0}\right)$ to be determined. The calculated values ranged from $1.63 \times 10^{-3} \mathrm{~cm} / \mathrm{s}$ for Au to $6.07 \times 10^{-4} \mathrm{~cm} / \mathrm{s}$ for Pt (Table 1). At room temperature, $k^{0}$ appears to be a good predictor for the Faradaic efficiencies of the group ten metals, with the highest rate constant observed for Pd and the lowest for Pt. The correlation between rate and efficiency is present for Au and GC as well. Koutecký-Levich analysis was repeated at a variety of temperatures, giving a value of $k^{0}$ for each. The Arrhenius relationship between rate and temperature is represented by the equation

$$
k=A e^{-E_{a} / R T}
$$

where $k$ is the rate constant, $A$ is a pre-exponential factor, $E_{a}$ is the apparent activation energy of the electrochemical reaction, $R$ is the universal gas constant and $T$ is the temperature. A representative Koutecky-Levich plot with a corresponding Arrhenius plot can be found in Figure 2 and its inset, respectively. The average activation energies for each electrode (listed in Table 1) generally show an inverse correlation with the Faradaic efficiencies previously calculated. From this, it is apparent that there is generally a higher selectivity for the oxidation of DBT in materials that have a lower barrier for the reaction. When looking at the group ten elements, there is a higher capacity for DBT oxidation moving from Ni to Pd, and then, interestingly, a decrease to Pt. The $\mathrm{Au}$ electrode, in comparison to the other materials tested, shows a greater selectivity for DBT oxidation under these electrolytic conditions, indicated by a notably higher faradaic efficiency, meaning more of the 
current passed during electrolysis contributes to DBT oxidation than other processes. This is supported by the calculated rate constant being the overall highest for all tested materials. For the symmetry coefficient, Values between 0.3 and 0.7 are typically observed, with lower values corresponding to sluggish kinetics. ${ }^{27}$ This, along with the higher activation energy is reflected in the low Faradaic efficiency observed for glassy carbon in the absence of water. The overall low measured efficiency for DBT consumption in this dry system suggests that there is a Faradaic loss in the reaction.

\section{Oxidation to Dibenzothiophene Sulfoxide}

The most common targets for DBT oxidation are $\mathrm{DBTO}$ or $\mathrm{DBTO}_{2}$. To better understand this oxidation pathway, we tested the above electrode materials in the presence of $2 \mathrm{M}$ water. Bulk electrolyses were carried out, resulting in the formation of both DBTO and DBT dimer as detected by LC-MS and NMR analysis (Fig. S5; S6, ESI). A commercial sample of DBTO was used to verify the spectral results, and a calibration curve was created to quantify the amount of DBTO present in post-electrolysis solutions (Fig. S7). Faradaic efficiencies for DBTO production were calculated and are listed in Table 2. The presence of water provides an alternate pathway via water oxidation, which offers a different energy barrier. To understand how these factors affect the activation energy of DBT oxidation, the same set of KouteckýLevich experiments were conducted as above. Ni and Pt electrodes were excluded due to water oxidation obscuring the sigmoidal DBT oxidation peaks. The calculated activation energies, rate, symmetry factor and overall percent conversion for DBT oxidation in the other materials are found in Table 2.

In the case of $\mathrm{Au}$ and $\mathrm{GC}$, there was a slight decrease in the measured activation energy, suggesting that the oxidation of DBT to DBTO has a lower barrier than that for DBT to its dimer. A dramatically higher Faradaic efficiency for DBT oxidation with water present was also observed for both electrodes, with GC more than doubling its value and Au reaching an impressive 87.9\%. This increase in DBT oxidation capacity is supported by the findings of Méndez-Albores et al., ${ }^{21}$ which saw an increase in current density for DBT oxidation to DBTO with a higher water concentration for 
GC working electrode. The same trend was not seen in the group ten metals, with no meaningful improvement to the Faradaic efficiencies measured for DBT oxidation, and an increase in the calculated activation energy for Pd. This indicates that the addition of water dramatically impacts the catalytic behaviour of the working electrode, and that the environment must be carefully considered when choosing an electrolytic system for DBT oxidation.

Looking at the overall conversion of DBT to its oxidation products, the selectivity of Au for DBTO is reflected in the relatively high percent conversion. While all the materials tested consumed similar amounts of DBT over the course of a one-hour electrolysis (30-40\%), the gold electrode oxidized over a quarter of the DBT to DBTO. GC had a similar selectivity to Au, with only a slight decrease in DBTO production, but the group ten metals did not produce nearly the same amount given the same amount of DBT being transformed. The DBT instead was oxidized to the dimer, or it was converted to a product that was not detected by our analytical methods. This demonstrates that the overall percent conversion does not give a complete picture of catalytic ability.

The dramatically higher Faradaic efficiency observed for Au in comparison to the other materials tested could be explained by the affinity that Au has for the sulfur atom in DBT. This proposed interaction has been investigated in the context of electronics, as shown by a study conducted by Tagami. In their work, a benzothiophene-based molecular wire and a gold electrode were modelled computationally using the extended-molecule approach. This system was assumed to have adsorption of sulfur from the benzothiophene wire to the gold electrode, forming a thiolate bond with a relatively long $\mathrm{S}$ - Au bond length of $2.55 \AA .^{28}$ Because dibenzothiophene is structurally analogous to the model molecule, we propose that a similar interaction is taking place between the Au electrode and DBT. The higher Faradaic efficiency could also be attributed to gold's resistance to catalyst poisoning in comparison to the rest of the set. In an experiment of DBT electrooxidation on metal nanoparticles supported on silica, it was found that, despite having a higher activation energy, gold was the most active nanoparticular catalyst when compared to platinum, palladium, and silver. ${ }^{29}$ This was explained by catalyst cycling, which revealed that platinum was more active than Au for the first cycle, then quickly died off, while gold maintained a consistent conversion. Of these two potential explanations, our data better supports the hypothesis of DBT interacting with the gold electrode surface. This phenomenon would create a local increase in concentration at the electrode interface. Assuming a constant diffusion coefficient of $2.30 \times 10^{-6} \mathrm{~cm}^{2} \mathrm{~s}^{-1},{ }^{30}$ we can use the limiting current density at various rotational rates and the equation:

$$
i_{\text {lim }}=\frac{n F D c^{0}}{\delta_{N}}
$$

to determine the effective concentration at the electrode surface, since the bulk concentration is the same 
for all solutions used. The results of this calculation show an apparent concentration for Au that is approximately double that of GC or Pd (Table S1).

\section{In Situ Activation Energy of Water Oxidation}

To understand the competition between the oxidation of DBT and water in our electrochemical system more completely, the activation energy for water oxidation for each electrocatalyst was determined under the experimental conditions. The Arrhenius relationship between temperature and catalytic activity can once again be employed to make this determination. Figure 3 shows a representative pair of plots that were used in the calculation of the Ea for water oxidation (Tafel plot and Arrhenius plot, Fig. S8). The resultant values are listed in Table 3. The group ten elements were found to have lower activation energies for water oxidation than $\mathrm{GC}$ or $\mathrm{Au}$, and the values calculated (approximately 30 $\mathrm{kJ} / \mathrm{mol}$ ) were in good agreement with reported water oxidation activation energies for catalysts containing these materials. ${ }^{31,32} \mathrm{Ni}$ and $\mathrm{Pt}$ had the lowest Ea and had an exponential increase in current density upon the application of an overpotential greater than $100 \mathrm{mV}$, which corresponds to water oxidation. This is a plausible explanation for why, in the presence of a higher concentration of water, the DBT oxidation reaction is outcompeted by oxidation of water by $\mathrm{Ni}$ and $\mathrm{Pt}$.

\section{Conclusions}

This study successfully classified a set of materials in terms of their electrocatalytic ability for the oxidation of DBT, in both the presence and absence of water. These results show that a low activation energy for DBT oxidation as well as a high barrier for water oxidation led to ideal catalytic performance, as demonstrated by gold which fulfilled both criteria and yielded a Faradaic efficiency of nearly $90 \%$ in our standard electrolytic conditions. Though there was not a direct correlation observed between period position of an element and the catalytic activity for DBT oxidation, our study illuminated the behaviour of these pure metals and glassy carbon, which are typically used as functionalized or polymetallic catalysts. These results are validated by the good agreement with literature values of similar reactions. The methods used to elucidate these kinetic parameters in our experiment can easily be applied to other materials in the future and will aid in catalyst design to optimize the DBT oxidation reaction, which will be a vital step in furthering oxidative desulfurization research.

\section{Acknowledgments}

This work was supported in part by an ACS Petroleum Research Fund Doctoral New Investigator Award (60013-DNI4). The authors also thank Binghamton University for start-up funding. Additional gratitude is extended to N. Dimitrov and E. Castillo for electrochemical supplies and experimental 
guidance.

\section{References}

1. A. Levy, E. Merryman and W. Reid, "Mechanisms of formation of sulfur oxides in combustion." Environ. Sci. Technol., 4, 653 (1970).

2. C. Pénard-Morand and I. Annesi-Maesano, "Air pollution: from sources of emissions to health effects." Breathe, 1, 109 (2004).

3. J. Summers and K. Barron, "The effects of $\mathrm{SO}_{2}$ on the performance of noble metal catalysts in automobile exhaust.” J. Catal., 57, 380 (1979).

4. Final Rule for Control of Air Pollution from Motor Vehicles, https://www.epa.gov/regulationsemissions-vehicles-and-engines, (accessed March 2021).

5. China: Fuel Implementation Schedule, https://dieselnet.com/standards/cn/fuel.php, (accessed May 2021).

6. T. Kabe, A. Ishihara and W. Qiang, Hydrodesulfurization and hydrodenitrogenation. Chemistry and Engineering, Wiley-VCH, 2000.

7. A. Safari and M. Vesali-Naseh, "Design and optimization of hydrodesulfurization process for liquefied petroleum gases." J. Clean. Prod., 220, 1255 (2019).

8. M. Houalla, N. Nag, V. Sapre, D. Broderick and B. Gates, "Hydrodesulfurization of dibenzothiophene catalyzed by sulfided $\mathrm{CoO}-\mathrm{MoO}_{3} \gamma-\mathrm{Al}_{2} \mathrm{O}_{3}$ : The reaction network." AIChE. J., 24, 1015 (1978).

9. T. Saleh, K. Sulaiman, S. Al-Hammadi, H. Dafalla and G. Danmaliki, "Adsorptive desulfurization of thiophene, benzothiophene and dibenzothiophene over activated carbon manganese oxide nanocomposite: with column system evaluation." J. Clean. Prod., 154, 401 (2017).

10. X. Zhou, X. Chen, Y. Jin and I. Markó, "Evidence of Two Key Intermediates Contributing to the Selectivity of P450 - Biomimetic Oxidation of Sulfides to Sulfoxides and Sulfones." Chem., Asian J., 7, 2253 (2012).

11. M. Hourani, "Desulfurization of thiophene by electrochemical perturbation." J. Electroanal. Chem., 368, 139 (1994).

12. F. Li, Y. Liu, Z. Sun, Y. Zhao, R. Liu, L. Chen and D. Zhao, "Photocatalytic oxidative desulfurization of dibenzothiophene under simulated sunlight irradiation with mixed-phase $\mathrm{Fe}_{2} \mathrm{O}_{3}$ prepared by solution combustion." Catal, Sci. Technol., 2, 1455 (2012).

13. R. Schucker, U.S. Patent, 6274026 B1, 2001.

14. S. Lalvani, M. Pata and R. Coughlin, "Sulphur removal from coal by electrolysis." Fuel, 62, 427 (1983).

15. H. Ahonen, WO Pat., 25636, 1993.

16. W. Wang, S. Wang, H. Liu and Z. Wang, "Desulfurization of gasoline by a new method of electrochemical catalytic oxidation." Fuel, 86, 2747 (2007).

17. G. Abdullah and Y. Xing, "Oxidation of dibenzothiophene in diesel with in situ produced hydrogen peroxide." Energy Fuels, 32, 8254 (2018).

18. Z. Aghbolagh, M. Khorrami and M. Rahmatyan, "Oxidative Desulfurization and Denitrogenation of Simulated Fuels Catalyzed by TBAPMo ${ }_{11} \mathrm{Cu} @ \mathrm{CuO}$ as a High-Performance and Recoverable Heterogeneous Phase-Transfer Catalyst." Energy Fuels, 34, 16366 (2020).

19. D. Zhao, Z. Sun, F. Li, R. Liu and H. Shan, "Oxidative Desulfurization of Thiophene Catalyzed by $\left(\mathrm{C}_{4} \mathrm{H}_{9}\right)_{4} \mathrm{NBr} \cdot 2 \mathrm{C}_{6} \mathrm{H}_{11} \mathrm{NO}$ Coordinated Ionic Liquid.” Energy \& Fuels, 22, 3065 (2008).

20 . C. Wang, W. S. Zhu, Y. H. Xu, H. Xu, M. Zhang, Y. H. Chao, S. Yin, H. M. Li and J. G. Wang, "Preparation of $\mathrm{TiO} 2 / \mathrm{g}-\mathrm{C} 3 \mathrm{~N} 4$ composites and their application in photocatalytic oxidative desulfurization." Ceram. Int., 40, 11627 (2014).

21. P. Zhang, L. Kang, M. Zhu and B. Dai, "Oxidative desulfurization catalyzed by a novel ZrP/MCM-41 catalyst with high performance." Sustain. Energy Fuels, 8, 4293 (2020).

22. O. Watts, "Rapid Nickel Plating." Trans. Electrochem. Soc., 29, 395 (1916). 
23. E. Méndez-Albores, M. González-Fuentes, M. Dávila-Jiménez and F. González, "Role of water in the formation of sulfoxide and sulfone derivatives during the electrochemical oxidation of dibenzothiophene in acetonitrile." J. Electroanal. Chem., 751, 7 (2015).

24. M. Pegis, J. Roberts, D. Wasylenko, E. Mader, A. Appel and J. Mayer, "Standard Reduction Potentials for Oxygen and Carbon Dioxide Couples in Acetonitrile and N,N-Dimethylformamide." Inorg. Chem., 54, 11883 (2015).

25. G. Bontempelli, F. Magno, G. Mazzocchin and S. Zecchin, "Cyclic and a.c. voltammetric study on dibenzothiophene in acetonitrile medium." J. Electroanal. Chem. Interf. Electrochem., 43, 377 (1973).

26. P. Audebert, J. Catel, G. Le Coustumer, V. Duchenet and P. Hapiot, "Electrochemistry and Polymerization Mechanisms of Thiophene-Pyrrole-Thiophene Oligomers and Terthiophenes. Experimental and Theoretical Modeling Studies." J. Phys. Chem., 102, 8661 (1998).

27. A. Bard and L. Faulkner, Electrochemical Methods, John Wiley \& Sons, Inc., New York, $2^{\text {nd }}$ edn, 2001 ch. 3, pp. 97-98.

28. K. Tagami, M. Tsukada, Y. Wada, T. Iwasaki and H. Nishide, "Electronic transport of benzothiophenebased chiral molecular solenoids studied by theoretical simulations." J. Chem. Phys., 119, 7491 (2003).

29. K. Castillo, Oxidation of Dibenzothiophene to Dibenzothiophene Sulfone Using Metal Nanoparticles Supported on Silica. Unpublished PhD Thesis, University of Texas, El Paso, (2010).

30. O. Dávila, L. Bergeron, M. Dávila Jiménez, I. Brillas, A. Roig Navarro, J. Beltrán Arandes, J. Sancho Llopis, "Study of the electrochemical oxidation of 4,6-dimethyldibenzothiophene on a BDD electrode employing different techniques." J. Electroanal. Chem., 894, 115364 (2021).

31. V. Vij, S. Sultan, A. Harzandi, A. Meena, J. Tiwari, N. Tiwari, W. Lee, T. Yoon and K. Kim, "NickelBased Electrocatalysts for Energy-Related Applications: Oxygen Reduction, Oxygen Evolution, and Hydrogen Evolution Reactions." Catal., 7, 7196 (2017).

32. A. Dokoutchaev, F. Abdelrazzaq, M. Thompson, J. Willson, C. Chang and A. Bocarsly, "Multicomponent Electrodes for Water Oxidation: From Combinatorial to Individual Electrode Study." Chem. Mater., 14, 3343 (2002). 


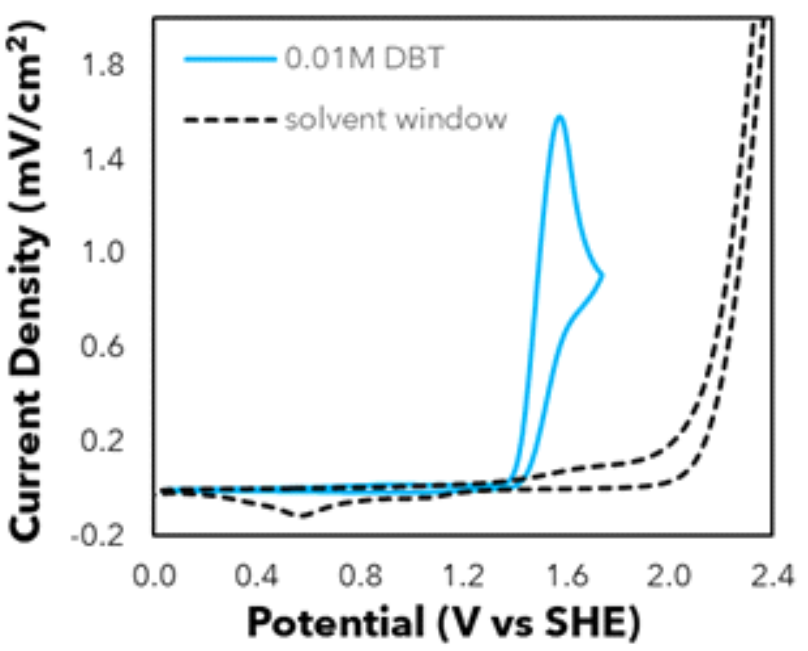

Figure 1. Cyclic voltammogram of $0.01 \mathrm{M}$ DBT in comparison to the solvent response in the same potential window, both measured using gold working electrode.

Table 1. Activation energy of each material tested in dry electrolytic conditions with the measured Faradaic efficiency, rate constant and symmetry coefficient at $298 \mathrm{~K}$.

\begin{tabular}{ccccc}
\hline Material & $\begin{array}{c}\text { Faradaic } \\
\text { Efficiency (\%) }\end{array}$ & $\begin{array}{c}\text { Activation Energy } \\
(\mathbf{k J} / \mathbf{m o l})\end{array}$ & $\boldsymbol{k}^{\mathbf{0}} \mathbf{( \mathbf { c m } / \mathbf { s } )}$ & $\boldsymbol{\alpha}$ \\
\hline $\mathrm{Au}$ & 56.5 & 9.49 & $1.63 \times 10^{-3} \pm 1.1 \times 10^{-4}$ & $0.43 \pm 0.08$ \\
$\mathrm{GC}$ & 18.4 & 26.4 & $1.09 \times 10^{-3} \pm 1.6 \times 10^{-4}$ & $0.28 \pm 0.01$ \\
$\mathrm{Ni}$ & 28.6 & 11.5 & $1.23 \times 10^{-3} \pm 5.8 \times 10^{-5}$ & $0.34 \pm 0.04$ \\
$\mathrm{Pd}$ & 30.2 & 7.55 & $1.31 \times 10^{-3} \pm 1.1 \times 10^{-4}$ & $0.31 \pm 0.02$ \\
$\mathrm{Pt}$ & 27.4 & 16.1 & $6.07 \times 10^{-4} \pm 5.8 \times 10^{-5}$ & $0.37 \pm 0.02$
\end{tabular}




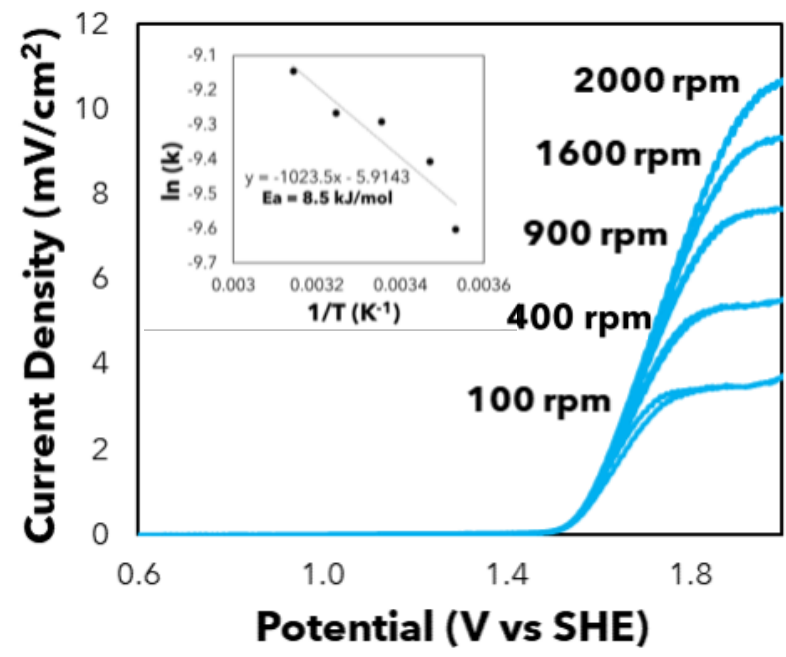

Figure 2. Koutecký-Levich plot of Pd electrode at 25 ${ }^{\circ} \mathrm{C}$ in dry conditions and the corresponding Arrhenius plot (inset).

Table 2. Activation energy of each material tested in $2 \mathrm{M}$ water with the measured Faradaic efficiencies, rate constants, symmetry factors, percent DBTO conversion and overall DBT consumption throughout electrolysis.

\begin{tabular}{ccccccc}
\hline Material & $\begin{array}{c}\text { Faradaic } \\
\text { Efficiency } \\
(\mathbf{\%})\end{array}$ & $\begin{array}{c}\text { Activation } \\
\text { Energy } \\
(\mathbf{k J} / \mathbf{m o l})\end{array}$ & $\boldsymbol{k}^{\mathbf{0}} \mathbf{( \mathbf { c m } / \mathbf { s } )}$ & $\boldsymbol{\alpha}$ & $\begin{array}{c}\text { DBTO } \\
\text { Conversion } \\
(\%)\end{array}$ & $\begin{array}{c}\text { DBT } \\
\text { Consumption } \\
(\%)\end{array}$ \\
\hline $\mathrm{Au}$ & 87.9 & 8.63 & $1.65 \times 10^{-3} \pm 1.3 \times 10^{-4}$ & $0.40 \pm 0.10$ & 25.6 & 40.0 \\
$\mathrm{GC}$ & 42.5 & 21.7 & $3.66 \times 10^{-4} \pm 1.2 \times 10^{-4}$ & $0.39 \pm 0.02$ & 20.7 & 44.0 \\
$\mathrm{Ni}$ & 28.5 & - & - & - & 6.45 & 39.0 \\
$\mathrm{Pd}$ & 30.6 & 18.3 & $8.65 \times 10^{-4} \pm 8.1 \times 10^{-4}$ & $0.37 \pm 0.08$ & 6.54 & 38.1 \\
$\mathrm{Pt}$ & 21.5 & - & - & - & 11.0 & 43.6
\end{tabular}



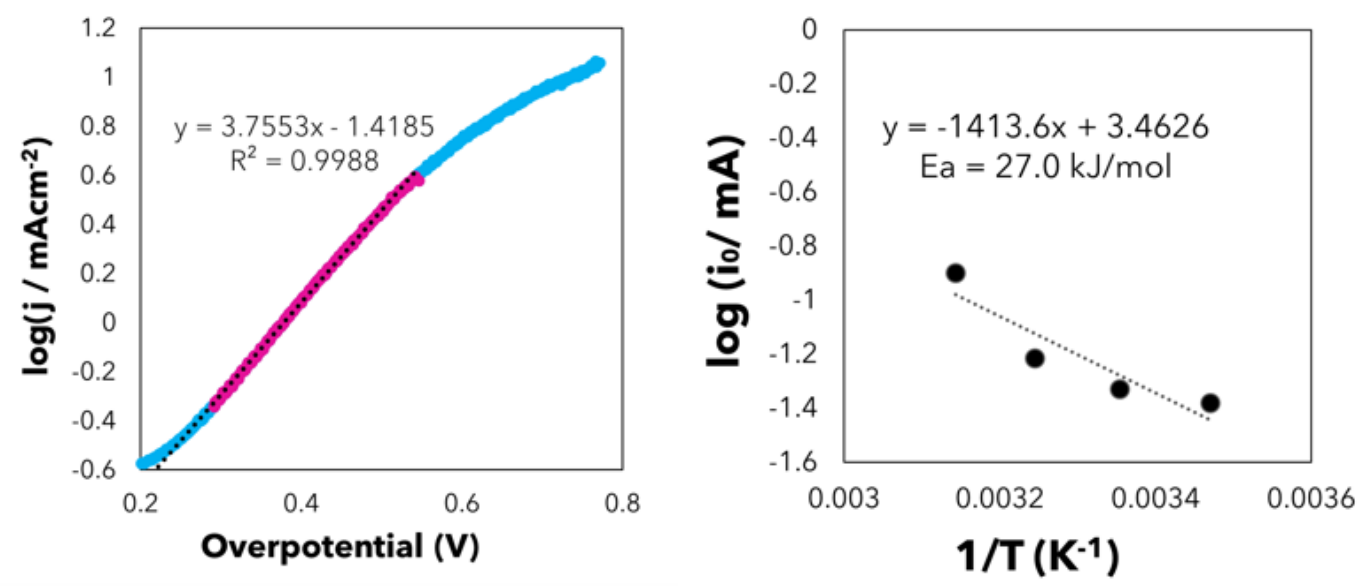

Figure 3. Tafel plot of $\mathrm{Pt}$ at $1500 \mathrm{rpm}$ at $25^{\circ} \mathrm{C}$ in $2 \mathrm{M}$ water and standard electrolyte solution (a) and the corresponding Arrhenius plot (b), showing an $E_{a}$ of $27.0 \mathrm{~kJ} / \mathrm{mol}$.

Table 3. Exchange current density at $25^{\circ} \mathrm{C}$ and activation energy of water oxidation at each electrode.

\begin{tabular}{ccc}
\hline Material & $\begin{array}{c}\text { Exchange Current Density }(- \\
\boldsymbol{l o g}\left[\mathbf{A} / \mathbf{c m}^{2}\right)\end{array}$ & $\begin{array}{c}\text { Activation Energy } \\
(\mathbf{k J} / \mathbf{m o l})\end{array}$ \\
\hline $\mathrm{Au}$ & 2.98 & 69.6 \\
$\mathrm{GC}$ & 0.893 & 58.3 \\
$\mathrm{Ni}$ & 0.331 & 27.4 \\
$\mathrm{Pd}$ & 1.22 & 33.4 \\
$\mathrm{Pt}$ & 1.33 & 27.0
\end{tabular}

